\title{
Feasibility Study of a Docosahexaenoic Acid-Optimized Nutraceutical Formulation on the Macular Levels of Lutein in a Healthy Mediterranean Population
}

\author{
Vicente Zanón-Moreno ${ }^{a, b}, c \quad$ Joan C. Domingo Pedrold \\ Silvia M. Sanz-González ${ }^{a, b} \quad$ Jorge Raga-Cervera ${ }^{a, e} \quad$ Juan Salazar-Corral ${ }^{b, f}$ \\ Maria Dolores Pinazo-Durán ${ }^{a, b}$ \\ aOphthalmic Research Unit "Santiago Grisolía” FISABIO, and Cellular and Molecular Ophthalmobiology Group of \\ the Department of Surgery at University of Valencia, Valencia, Spain; 'bpanish Network of Cooperative Research \\ in Ophthalmology (OFTARED), Carlos III Health Institute, Ministry of Science, Innovation and Universities, Madrid, \\ Spain; ${ }^{C}$ Faculty of Health Sciences, Valencian International University, Valencia, Spain; ${ }^{\mathrm{d}}$ Department of Biochemistry \\ and Molecular Biomedicine at the Faculty of Biology, University of Barcelona, Barcelona, Spain; 'Department of \\ Ophthalmology, Hospital of Manises, Manises, Spain; fInstitute of Ophthalmic Research "Ramón Castroviejo", \\ Complutensis University of Madrid, Madrid, Spain
}

\section{Keywords}

Macular pigment optical density · Lutein · Docosahexaenoic acid · Ocular health

\begin{abstract}
Introduction: Macular pigment optical density (MPOD) plays a pivotal role in maintaining macular structure and functioning. Research shows that daily consumption of lutein reduces the risk of eye diseases such as age-related macular degeneration. Objective: This study analyzes the influence of a supplementation containing lutein and antioxidant vitamins either with or without docosahexaenoic acid (DHA), with the main objective of identifying MPOD changes in both eyes at the end of the follow-up using the Visucam ${ }^{\circledR}$ retinograph. The secondary end point was to determine variation in the lutein and DHA levels in plasma and red blood cell membranes (RBCMs), respectively. Methods: One hundred healthy participants (200 eyes) aged $40-70$ years (mean age 49.3 years, $S E M=13.7$ ) were randomized in a 1:1 ratio to receive daily one of the following
\end{abstract}

karger@karger.com www.karger.com/ore

Karger $\stackrel{\text { ' }}{5}$
(C) 2020 The Author(s)

Published by S. Karger AG, Basel

This is an Open Access article licensed under the Creative Common Attribution-NonCommercial-4.0 International License (CC BY-NC) (http://www.karger.com/Services/OpenAccessLicense), applicable to the online version of the article only. Usage and distribution for commercial purposes requires written permission. supplements for 3 months: lutein group (LT-G, $n=49$ ) and lutein plus DHA group (LT/DHA-G, $n=51$ ). The MPOD was measured at baseline and end of the follow-up by retinography (Visucam ${ }^{\circledR}$ retinograph). Lutein in plasma was determined by HPLC, and DHA in RBC membranes was analyzed by gas chromatograph/mass spectrometer. Results: From baseline, MPOD showed significantly higher values in the LT/DHA-G than in the LT-G at the end of the study $(p<0.0001)$. Significantly higher lutein in plasma $(p<0.0001)$ and DHA $(p<0.0001)$ levels in the RBC membrane were seen in the LT/DHA-G than in the LT-G at the 3-month follow-up. Conclusion: Lutein supplementation improves MPOD in healthy subjects from a Mediterranean population being significantly increased in the presence of DHA. Therefore, our findings highlight the relevance of the adjunctive role of DHA for better lutein availability.

(C) 2020 The Author(s)

Published by S. Karger AG, Basel

Sharing first authorship: V.Z.-M. and J.C.D.P.

Group leaders equally sharing the last authorship: J.S.-C. and M.D.P.-D. 


\section{Introduction}

Several modifiable factors including nutrition have been studied in connection to the pathogenic mechanisms of eye diseases such as dry eyes, cataracts, glaucoma, and age-related macular degeneration (AMD) [1]. Vitamins, minerals, carotenoids, and long-chain polyunsaturated fatty acids (LC-PUFAs) have been studied in connection to ocular physiopathology [2], with some interesting results which will be discussed further.

The role of trace elements on eye diseases has been widely studied. A high copper concentration has been reported in plasma samples from patients with diabetic retinopathy, compared to control subjects [3]. In another study conducted on tear samples, Vinetskaia and Iomdina [4] observed alterations in the levels of iron, magnesium, and aluminum and, to a lesser extent, in the levels of copper and zinc in diabetic retinopathy patients. Instead, they found lower levels of zinc and higher levels of iron in tears of open-angle glaucoma patients. Decreased zinc levels in the retina have also been associated with the onset and progression of age-related eye diseases [5].

Carotenoids (also known as tetraterpenoids) are organic pigmented molecules that are produced by a wide variety of fruits (mango and papaya), vegetables (carrots and pumpkins), eggs (yolk), birds (pheasant, toucan, and parrot), seafood (lobster, salmon, and shrimp), and spices (saffron and paprika), which give these foods their yellow, orange, or red color. Leafy vegetables such as broccoli, kale, and spinach also contain high amounts of carotenoids. The major classes of carotenoids include the xanthophylls such as lutein (LT) and zeaxanthin (ZX) and carotenes ( $\alpha$-carotene, $\beta$-carotene, and lycopene). The human body does not synthesize any of these carotenoids [6]. Interestingly, White et al. [7] have recently reported that availability of LT in food (or pills) noticeably increases when consumed together with fat. Specific xanthophylls are particularly located in the central retina, constituting the macular pigment. LT is the predominant xanthophyll in the retina, ZX in the macular periphery, and LT-derived meso-ZX in the foveal area [8]. All of them play a pivotal role in maintaining the morphology and function of this structure [9]. Several studies have analyzed the role of macular pigment optical density (MPOD) in ocular health and disease, finding that low MPOD increases the risk of macular diseases such as $A M D$, while high MPOD protects the eyes against a variety of pathologies [10-12].

Among the omega-3 ( $\omega-3)$ LC-PUFAs, the dietary precursor linolenic acid (ALA, C18:3) is transformed with dif- ficulties to both eicosapentaenoic acid (EPA; C20:5 $\omega 3$ ) and docosahexaenoic acid (DHA; C22:6 63 ), which are the major LC-PUFAs active types in humans. The retina is the human tissue that contains the highest lipid profiles enriched in DHA LC-PUFA, which plays a pivotal role in the regulation of retinal morphology and functioning [13]. A variety of reports have shown that $\omega$-3 LC-PUFAs supplementation (from food, as well as from oral capsules) induces positive responses on global health [14] and on pathologic situations including degenerative and aging-related disorders [15]. Long-chain omega-3 (LC $\omega$-3) fatty acids are incorporated into the cell membrane phospholipids. Harris reported that the $\omega-3$ index measures the percentage of the $\omega-3$ LC-PUFAs (EPA + DHA) in relation to total red blood cell membranes (RBCMs) fatty acids [16], so the RBCMs have been proposed as a precise marker of dietary fatty acid intake [17]. Epidemiological and experimental studies have demonstrated that the RBCM EPA and EPA + DHA (as markers of $\omega-3$ dietary PUFA status) were closely related with neovascular AMD [18].

Previous studies reported that MPOD did not change significantly when LT and DHA were administered together [19]. In fact, the authors argued that only LT and DHA worked synergistically to improve the entire MPOD spatial profile [19].

Summing up the current scientific evidence and taking into account the importance of xanthophylls for vision, we designed this study to analyze the effects of an optimized nutraceutical formulation regime over 3 months, based on a high DHA triglyceride content. We have determined the DHA variation in the RBCMs and the plasma and macular levels of LT in a healthy adult Mediterranean population.

\section{Materials and Methods}

The present work adheres to the Ethical Principles for Medical Research Involving Human Subjects (Declaration of Helsinki [Edinburgh, 2000]), the Ethics Committee standards of the study centers, and the Spanish Agency of Medicines and Medical Devices (AEMPS). Signed informed consent was obtained from all the participants. EudraCT number 2019-002356-16 for the sponsor's protocol code number $36 / 16$.

\section{Study Design}

A prospective interventional multicenter double-masked study was conducted between 2016 and 17/2017 and 18 in the Mediterranean area of Valencia (Spain). The main purpose was to evaluate the effect on MPOD of 2 commercial supplements having a similar formulation relating vitamins and minerals, close to the one used in the Age-Related Eye Disease Study (AREDS). The only difference between them was the presence of LT (formula 1) or LT + 
Table 1. Inclusion and exclusion criteria for the study participants

\begin{tabular}{ll}
\hline Inclusion criteria & Exclusion criteria \\
\hline $\begin{array}{l}\text { Individuals of both sexes } \\
\text { Aged } 40-70 \text { years }\end{array}$ & Aged $<40$ or $>70$ years \\
\hline Healthy well-nourished persons & $\begin{array}{l}\text { Dietary or alimentary disorders or having relevant ocular or systemic } \\
\text { diseases or treatments }\end{array}$ \\
\hline $\begin{array}{l}\text { Able to understand the study goals and to provide signed } \\
\text { informed consent }\end{array}$ & Unable to understand and to participate in the study \\
\hline
\end{tabular}

Table 2. Characteristics of both study formulas for the oral supplementation

\begin{tabular}{|c|c|c|c|c|}
\hline \multirow[t]{2}{*}{$\omega-3$ LC-PUFA } & \multicolumn{2}{|c|}{$\begin{array}{l}\text { Supplement containing } \\
\text { LT formula } 1\end{array}$} & \multicolumn{2}{|c|}{$\begin{array}{l}\text { Supplement containing } \\
\text { LT/DHA formula } 2\end{array}$} \\
\hline & dose/day & $\% \mathrm{RI}$ & dose/day & $\% \mathrm{RI}$ \\
\hline DHA, mg & 0 & & 700 & \\
\hline EPA, mg & 0 & & 85 & \\
\hline DPA, mg & 0 & & 60 & \\
\hline \multicolumn{5}{|l|}{ Vitamins } \\
\hline $\mathrm{C}, \mathrm{mg}$ & 80 & 100 & 53.4 & 66 \\
\hline $\mathrm{E}, \mathrm{mg}$ a-TE & 12 & 100 & 8 & 66 \\
\hline $\mathrm{B} 1, \mathrm{mg}$ & 1.1 & 100 & 0.74 & 66 \\
\hline $\mathrm{B} 2, \mathrm{mg}$ & 1.4 & 100 & 0.94 & 66 \\
\hline $\mathrm{B} 3, \mathrm{mg}$ & 16 & 100 & 10.6 & 66 \\
\hline $\mathrm{B} 6, \mathrm{mg}$ & 1.4 & 100 & 0.94 & 66 \\
\hline $\mathrm{B} 9, \mu \mathrm{g}$ & 200 & 100 & 133.4 & 66 \\
\hline $\mathrm{B} 12, \mu \mathrm{g}$ & 2.5 & 100 & 1.66 & 66 \\
\hline \multicolumn{5}{|l|}{ Minerals } \\
\hline $\mathrm{Zn}, \mathrm{mg}$ & 7.5 & 75 & 3.32 & 33 \\
\hline $\mathrm{Cu}, \mathrm{mg}$ & 1 & 100 & 0.32 & 33 \\
\hline $\mathrm{Se}, \mu \mathrm{g}$ & 55 & 100 & 18.32 & 33 \\
\hline $\mathrm{Mn}, \mathrm{mg}$ & 2 & 100 & 0.66 & 33 \\
\hline \multicolumn{5}{|l|}{ Others } \\
\hline Lutein, mg & 6 & - & 6 & - \\
\hline $\mathrm{ZX}, \mathrm{mg}$ & 0.3 & - & 0.6 & - \\
\hline Glutathione, mg & 1 & - & 4 & - \\
\hline
\end{tabular}

$\% R I$, percentage of the reference intake; LT, lutein; LT/DHA, LT/docosahexaenoic acid; EPA, eicosapentaenoic acid; DPA, docosapentaenoic acid; $\omega$-3, omega-3; ZX, zeaxanthin.
DHA (formula 2) given in a subject-masked fashion over 3 consecutive months, as measured by the Visucam ${ }^{\circledR}$ retinograph. The secondary end point was to look at the effects of both supplement regimes on the plasma levels of LT and DHA in the RBCM, via standard laboratory assays.

Patient Management

For this study, 125 healthy potential participants were initially selected in ophthalmologic appointments during an ordinary visit in the study centers. These initially potential participants were interviewed according to the inclusion/exclusion criteria (Table 1), extensively informed about the study characteristics and provided written informed consent for participation in the study. Briefly, the following baseline interview data were recorded: sociodemographics, personal/familial background, and lifestyle (nutrition). At the first visit, patients underwent a systematized ophthalmic examination in both eyes including best-corrected visual acuity (BCVA), intraocular pressure (IOP), and ocular fundus examination and/or retinographies. Of the potential participants, 110 suitable participants aged 40-70 years were recruited and homogeneously randomized in a 1:1 ratio to receive the following treatments for 3 months: lutein group (LT-G, $n=55$ ): formula 1, dosing $6 \mathrm{mg}$ of LT per day; or LT plus DHA group (LT/DHA-G, $n=55$ ): formula 2 , dosing $6 \mathrm{mg}$ of LT and $700 \mathrm{mg}$ of a concentrated DHA triglyceride 


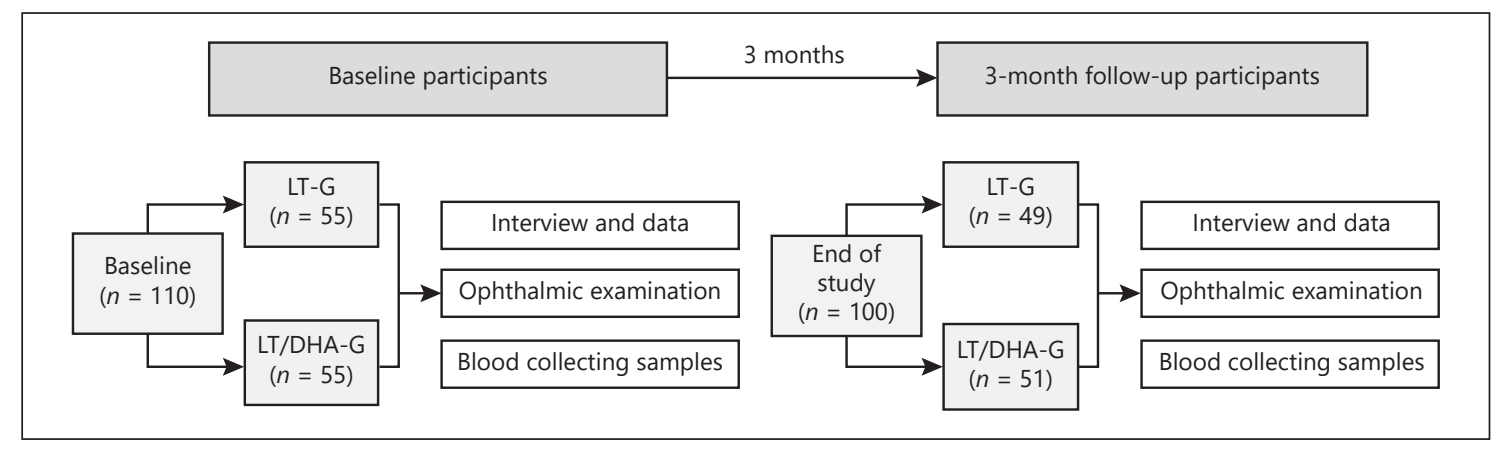

Fig. 1. Flowchart showing classification and operative definition of the study participants. LT-G, lutein group (6 mg LT daily); LT/DHA-G, LT/docosahexaenoic acid group (6 mg LT and $700 \mathrm{mg}$ DHA daily).

daily (Table 2). This is a DHA triglyceride which has a high antioxidant activity patented to prevent cellular oxidative damage [2022]. Both food supplements were commercial formulations gently provided by the pharmaceutical company in similar boxes to the investigators in a masked fashion (BrudyRetina, box A and BrudyRetina box B; Brudylab, SL, Barcelona, Spain). Neither the investigators nor the participants knew the specific composition of the 2 formulas. The commercialized nutraceuticals were given to the participants without any charge. Blood samples were collected in fasting condition to analyze the plasma levels of LT and the RBCM DHA content by high-performance liquid chromatography and gas chromatography (GC), respectively. At the end of the 3-month supplementation period, the participants were visited again to perform the same protocol, as reflected in the Figure 1.

Ophthalmic Examination

Eye examination protocol in each eye included BCVA, IOP, and ocular fundus inspection/photographs (Visucam ${ }^{\circledR} 200$ pigment module; Carl Zeiss Meditec Iberia, Madrid, Spain). Each patient was seated and conveniently positioned for ocular examination under mesopic illumination. Three retinographies were taken consecutively from each eye at both study points according to the manufacturer's instructions. Retinographies were evaluated to obtain optimal quality for MPOD determinations according to previous reports $[23,24]$. Augmentation of the relative densitometric units from baseline of the retinographs and their corresponding data from each eye were considered improvement, and the results were recorded as percentages.

Sampling Protocols

Blood sampling was scheduled at baseline and 3-month followup for biochemical assays. Antecubital vein blood was collected under fasting conditions at 8:00 a.m. from each participant. Tubes were centrifuged at 3,000 rpm for $10 \mathrm{~min}$, and the plasma/erythrocytes were separated. Aliquots of the upper (plasmatic layer) and lower (erythrocytic layer) fractions from each participant were placed in separate Eppendorf tubes, labeled and stored at $-80^{\circ} \mathrm{C}$ until processing. All experiments were performed in duplicate, and the protocols are detailed below.

\section{Laboratory Assays}

LT analysis in plasma was obtained by using the modified method of Colmán-Martínez [25] extraction, and determination of LT from the plasma samples was performed in twilight to avoid oxidation of the compounds. Plasma aliquots were thawed and protein was precipitated, which was followed by extraction with hexane. The extraction process was repeated twice more. The supernatants were dissolved in methyl tert-butyl ether (MTBE). Chromatographic analysis was carried out in a Waters 2795 Alliance HT highperformance liquid chromatography system coupled to a Waters 2996 photodiode array detector (Waters Chromatography Corp., Milford, MA, USA). Chromatographic separation was performed on a reversed-phase column YMC (YMC America Inc., Allentown, PA, USA) at room temperature. The mobile phase consisted of a linear gradient of methanol, MTBE, and water. The gradient consisted of $6 \% \mathrm{MTBE}$ to $56 \%$ linear gradient in $20 \mathrm{~min}$ and then changed to $90 \%$ MTBE and was kept for 2 min and returned to initial conditions. The water was kept constant at $4 \%$. The flow rate throughout the run was $0.6 \mathrm{~mL} / \mathrm{min}$. The diode array detector monitored the peaks at a range of $220-600 \mathrm{~nm}$. The integration was performed with Waters ${ }^{\circledR}$ Empower software. LT was identified according to retention time and absorption spectra at $450 \mathrm{~nm}$. The concentration in the samples was calculated using the LT standard curve. Fatty acid composition in RBCM was determined as methyl esters after methylation reaction, using the method of Lepage and Roy [26]. GC analysis was performed on a Shimadzu GCMSQP2010 plus gas chromatograph/mass spectrometer (Shimadzu, Kyoto, Japan), with a Shimadzu AOC-20i autoinjector, and a Shimadzu AOC-20 s autosampler. The column used was a Suprawax-280 (Teknokroma, Barcelona, Spain). Operation conditions were as follows: the injector was used in splitless mode, and the temperature was kept at $250^{\circ} \mathrm{C}$. The temperature program was as follows: initial, $150^{\circ} \mathrm{C}$ with a $0.25 \mathrm{~min}$ hold, ramp at $35^{\circ} \mathrm{C} / \mathrm{min}$ to $200^{\circ} \mathrm{C}, 8^{\circ} \mathrm{C} / \mathrm{min}$ a $225^{\circ} \mathrm{C}$ with a $3.2 \mathrm{~min}$ hold, and then $80^{\circ} \mathrm{C} / \mathrm{min}$ to $245^{\circ} \mathrm{C}$ with a $2.75 \mathrm{~min}$ hold. Helium was used as the carrier gas. The interface and ion source temperatures were 255 and $200^{\circ} \mathrm{C}$, respectively. The MS ionization mode was electron ionization. Fatty acid methyl esters (FAMEs) were identified through mass spectrometry and through comparison of the elution pattern and relative retention times of FAME with the reference FAME mixture (GLC-744 $\mathrm{Nu}$-Chek Prep. Inc., Elysian MN, USA). The results were expressed in relative amounts (percentage molar of total fatty acids).

\section{Statistical Analysis}

The sample size was determined using the freeware GRANMO sample size calculation program v.7.12 (https://www.imim. 
cat/ofertadeserveis/software-public/granmo, Institut Hospital del Mar d'Investigacions Mèdiques, Barcelona, Spain). Accepting an alpha risk of 0.05 and a beta risk of 0.2 in a 2-sided test, 64 subjects are necessary in first group and 64 in the second to find as statistically significant a proportion difference, expected to be of 0.5 in group 1 and 0.75 in group 2 . It has been anticipated a dropout rate of $10 \%$. The ARCSINUS approximation was used to carry out these calculations. Microsoft Excel and IBM Statistical Package for the Social Sciences (SPSS) V.24.0 programs (SPSS Inc., Chicago, IL, USA) were used. For continuous variables, nonparametric (Mann-Whitney U) and parametric ( $t$ test) statistics were utilized, and the results were expressed as mean \pm standard error of the mean (SEM). Categorical variables were expressed as percentages. A $p$ value $<0.05$ was considered statistically significant, which was adjusted by Bonferroni correction when pairwise comparison in multiple groups was conducted. Correlation analysis was used to evaluate the association between the MPOD levels at baseline and the changes throughout the interventional study.

\section{Results}

\section{Demographics}

The final number of participants was 100 (49 assigned to the LT-G and 51 assigned to the LT/DHA-G), and 200 eyes were analyzed. The rate of withdrawal was $10 \%$, and withdrawals were due to deterioration of health, lack of motivation, confusing ocular fundus retinographs, and blood samples failing to be processed or showing confusing data.

The mean age of the LT-G was $44.6 \pm 13.9$ versus 44.0 \pm 13.5 years for the LT/DHA-G. The range of age of the participants is narrow enough to avoid an impact on the density of the crystalline lens, to prevent a confounding on the Visucam measurements. Distribution by gender was $51.1 \%$ men and $48.9 \%$ women in the LT-G and $40.8 \%$ men and 59.2\% women in the LT/DHA-G.

\section{Ophthalmic Examination}

No changes in the BCVA and IOP values between groups were observed between the 2 study points. However, values of the MPOD were significantly higher at the 3-month follow-up than baseline in the eyes of both participant groups (Fig. 2). Furthermore, there were noticeably higher values in the LT/DHA-G eyes (Fig. 2b) than in the LT-G (Fig. 2a). The eyes of the LT-G showed a $27.5 \%$ increase in the right eye and a $32.2 \%$ in the left eye for MPOD, with a global increase of $29.0 \%$ in both eyes. The eyes of the LT/DHA-G showed an increase of $38.5 \%$ in the right eye and $40.6 \%$ in the left eye for MPOD, with a global increase of $39.6 \%$ in both eyes. However, 11 participants in the LT-G and 5 participants in the LT/DHA-

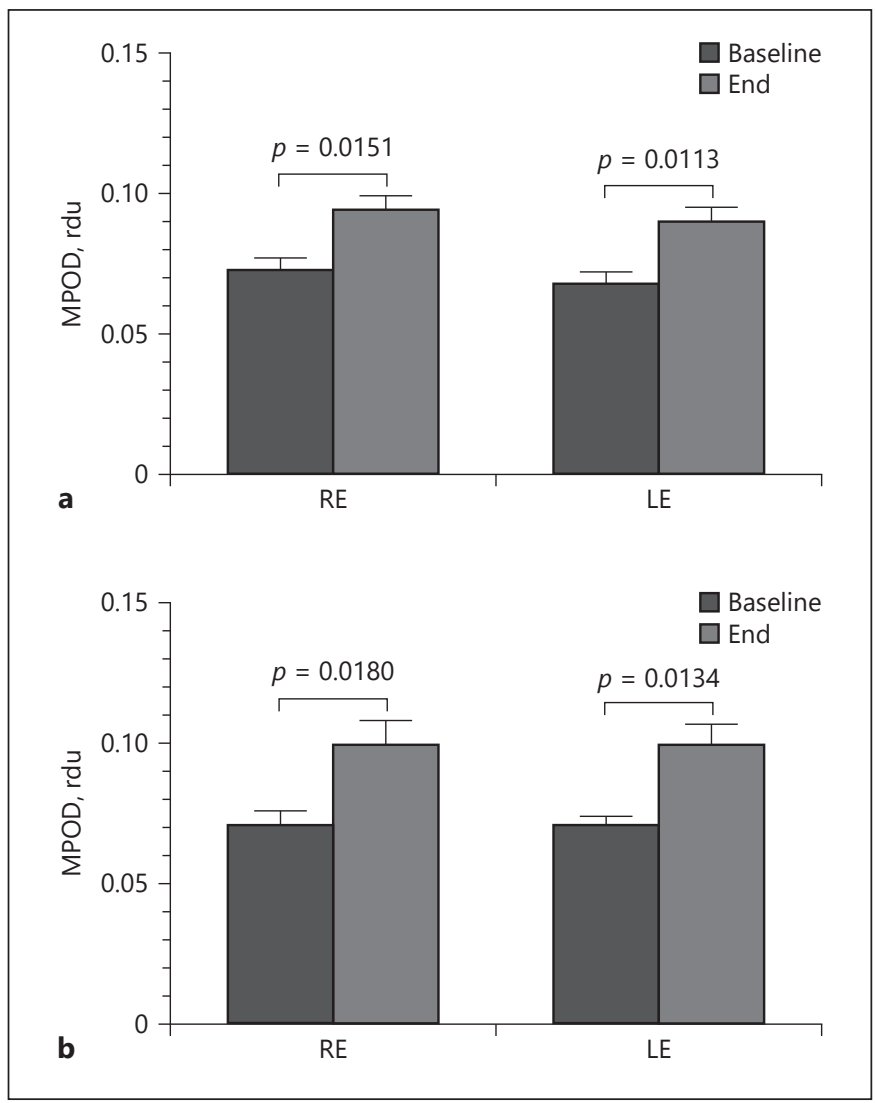

Fig. 2. Basal and final MPOD of the right and left eyes of the LT-G (a) and of the LT/DHA-G (b). Data represents mean \pm SEM $(n=$ 47 in LT-G; $n=49$ in LT/DHA-G). MPOD, macular pigment optical density; rdu, relative densitometric units; RE, right eye; LE, left eye; LT-G, lutein group; LT/DHA-G, LT/docosahexaenoic acid group.

G showed less than a $10 \%$ increase in MPOD concentration at the end of the study.

\section{Laboratory Assays}

The biochemical analyses demonstrated a statistically significant augmentation in plasma LT in the LT/DHA-G compared to the LT-G after 3 months of the supplement regime (Fig. 3a). Moreover, significantly higher RBCM DHA levels were seen in the LT/DHA-G compared to the LT-G at the end of the study (Fig. 3b).

As shown in Figure $4 \mathrm{a}$ (LT-G) and 4b (LT/DHA-G), the MPOD showed a near-significant positive correlation with plasma LT in the group supplemented with formula 2 (LT/DHA-G, $r=0.291, p=0.085$ ), but not in the group supplemented with formula 1 (LT-G, $r=0.014, p=0.941$ ). Because MPOD correlated positively with LT plasma 

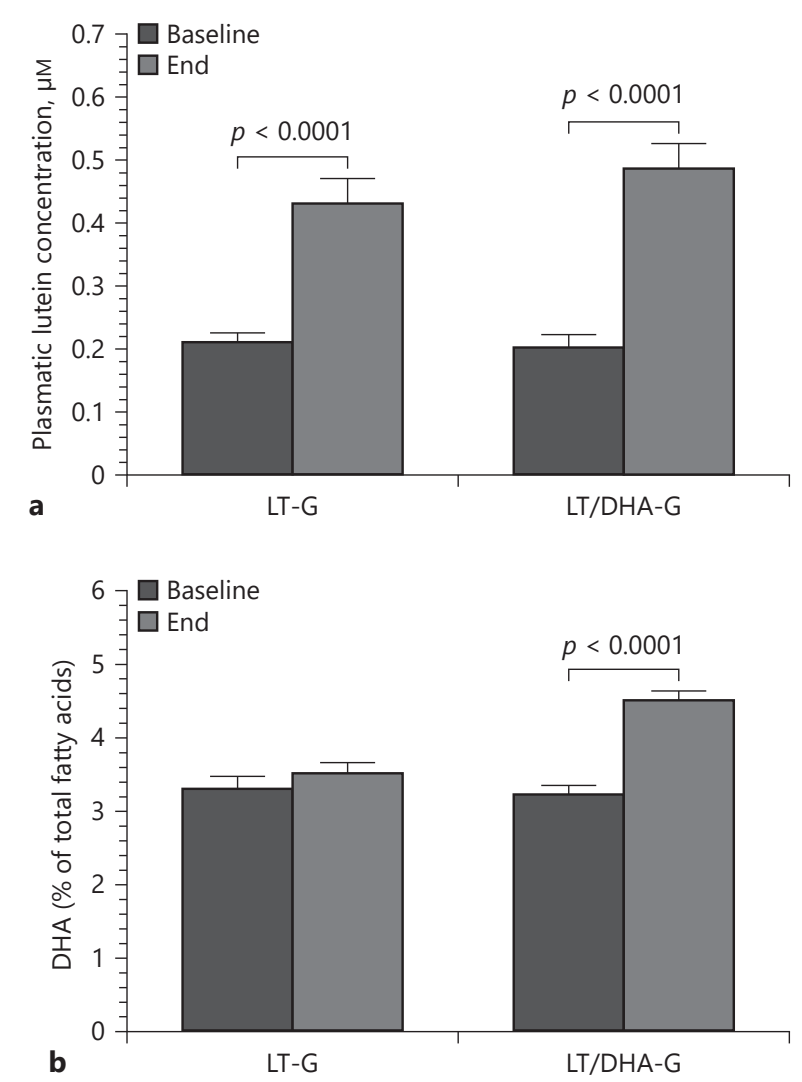

Fig. 3. Basal and final plasmatic LT concentrations (a) and RBCM DHA content (b) of both study groups. LT concentration was measured by HPLC analysis and DHA content was determined by GC/ mass spectrometry analysis. Data represents mean \pm SEM $(n=38$ in LT-G; $n=36$ in LT/DHA-G). LT-G, lutein group; LT/DHA-G, LT/docosahexaenoic acid group; RBCM, RBC membrane; GC, gas chromatography.

concentrations in the LT/DHA-G and this increased with the addition of DHA in the nutraceutical formulation, we compared the correlation coefficients of the MPOD and the RBCM DHA content of the total study population. There is a positive correlation of RBCM DHA content with MPOD and was statistically significant $(r=0.244$, $p=0.0037$ ) (Fig. 4c).

\section{Discussion}

We evaluated the effects of 2 oral supplements dosing the same daily amount of LT (in formula 1) and LT/DHA (in formula 2) on the MPOD in a healthy population. At

Optimized Nutraceutical Formulation with DHA on Macular Levels of Lutein
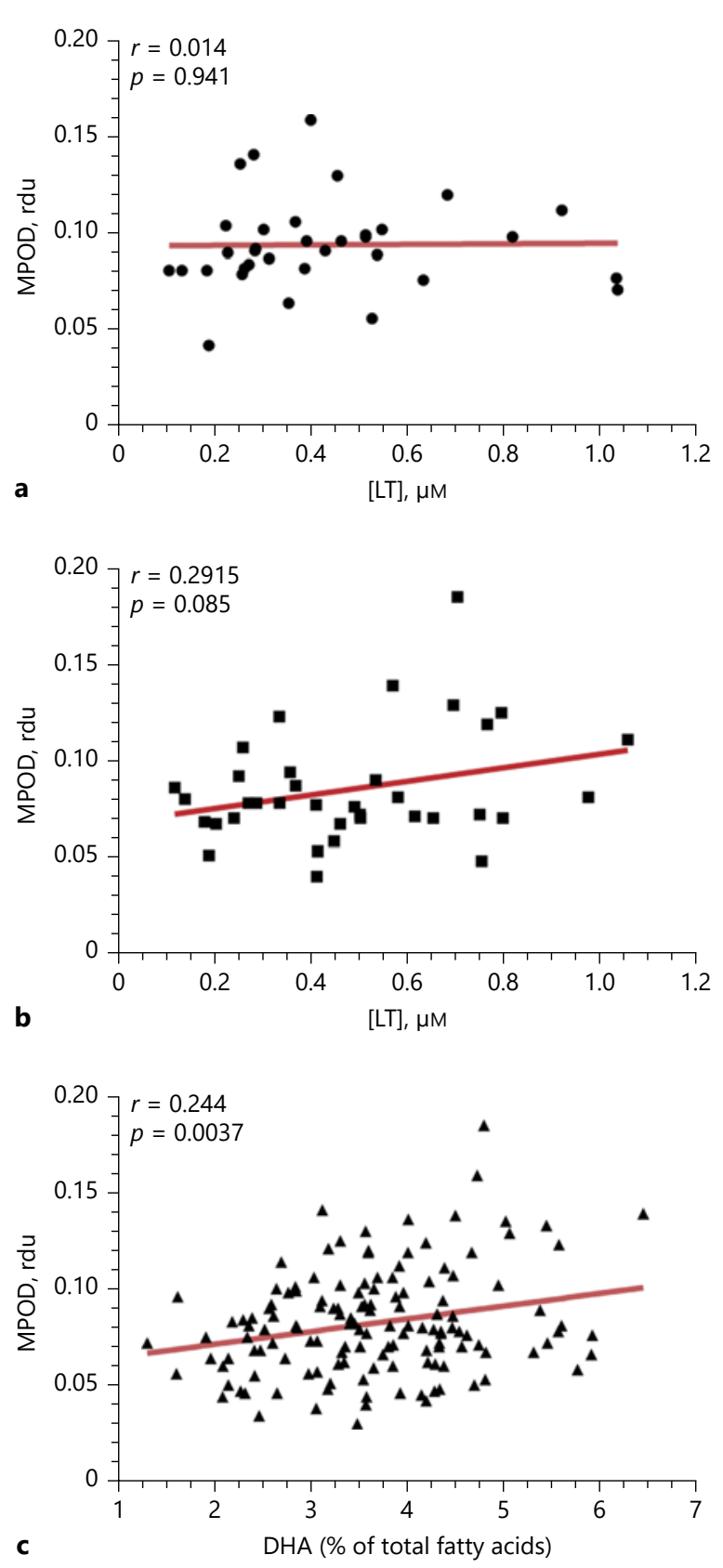

Fig. 4. Scatterplots depicting the correlation between MPOD with the plasmatic LT concentration in the LT-G $(\mathbf{a}, n=38)$ and in the LT/DHA-G (b, $n=36)$. Correlation between MPOD with RBCM DHA levels including all the population studied is shown (c, $n=$ 140). MPOD, macular pigment optical density; rdu, relative densitometric units; LT-G, lutein group; LT/DHA-G, LT/docosahexaenoic acid group; RBCM, RBC membranes.

Ophthalmic Res 2021;64:1068-1076 DOI: $10.1159 / 000509439$ 
3-month follow-up, we found a significant increase in the MPOD levels in the participants randomly assigned to formula 2 (LT/DHA-G) compared to formula 1 (LT-G) $(p<0.0001)$. Moreover, significantly higher plasma LT $(p<0.0001)$ and DHA RBCM levels $(p<0.0001)$ was seen in the LT/DHA-G compared to the LT-G. Our data strongly indicate that nutraceuticals containing LT noticeably improve MPOD in healthy subjects, but with a significant increased dose-dependent response in the presence of DHA in triglyceride form.

Studies similar to ours have also reported that the LT macular concentration is a modifiable nutritional factor in health and disease [12, 27-31]. However, other authors did not find any relationship between the nutritional intervention and changes in the LT macular levels, as in the work by Johnson et al. [19], which was carried out in a sample of 49 elder women without maculopathy. The MPOD did not change when LT and DHA were administered together. More recently, Korobelnik et al. [24] have suggested that patients with neovascular AMD did not show modified MPOD levels after 6 months of LT and ZX dietary supplementation, despite their plasma levels clearly showing continuous exposure to these xanthophylls. Olmedilla-Alonso et al. [32] also reported that neither the xanthophylls, the anthocyanins, nor a combination of these 2 (at the selected doses) were capable of increasing MPOD after 8 months of supplementation in postmenopausal women. Differences in the study design, the formulation, as well as in the device used for analyzing the MPOD may be contributing to the difficulty in comparing between studies.

An important result of the present work is that DHA in triglyceride form reinforces the transport and uptake of LT into the macula, probably by inducing changes in the lipoprotein profile that, in turn, favors the availability of xanthophylls in the macula. We have confirmed the correlation between LT plasma levels and MPOD only in the LT/DHA-G, while no correlation was found in the LT-G. Among the different determinants of MPOD considered, $\omega$-3 LC-PUFAs dietary intake, especially with DHA, has been proposed as a key factor $[19,33]$. These studies have shown that in total plasma phospholipids, analysis of the association between the DHA level and MOPD were not significantly correlated. Contrary to plasma DHA level as a valid biomarker for LC-PUFAs dietary intake, by analyzing the population of both treatment groups (pre- and posttreatment), our study shows a significant positive relationship between RBCM DHA levels and MPOD. This positive correlation suggests an argument about the status of DHA within the retina and its role in relation to macular pigment concentration.
It has also been observed that some of our study participants (11\% of the LT/DHA-G and $17 \%$ of the LT-G) displayed unnoticeable MPOD changes, despite having significantly higher plasmatic LT and RBCM DHA levels with respect to baseline (see Fig. 2, 3, 4). However, the proportion of nonresponse was lower among individuals assigned to formula 2 than formula 1 and appreciably lower than those reported by other authors in normal and pathologic eyes [34-36]. Interestingly, Obana et al. [37] have precisely described 3 types of response to administration regarding the increase in MPOD and serum LT levels in a sample of 36 healthy participants: retinal responders (with an increase of both MPOD and serum LT levels), retinal nonresponders (neither MPOD nor serum LT concentration increases, but no MPOD changes), and retinal and serum nonresponders.

The inter-individual variability in the absorption, circulation, and tissue responses of dietary carotenoids is widely accepted. Recent discoveries in several proteins involved in human carotenoid metabolism point to the fact that genetic variants in genes encoded for these proteins may affect their expression and subsequently the carotenoid availability and function [38]. Strong evidence indicates that gene variants related to carotenoid metabolism play a pivotal role in the uptake of LT and ZX into the macula. Feigl et al. [39] demonstrated in 20 healthy participants that MPOD levels can be related to high and low $\beta$-carotene conversion beta-carotene monooxygenase and that the above effect may be different in pathologic retinas. Through a study of carotenoids in age-related eye disease involving 1,585 women, Meyers et al. [40] concluded that MPOD is a multifactorial phenotype associated with variation in genes related to carotenoid transport, uptake, and metabolism that is independent of the well-known dietary and lifestyle influences on MPOD. In this scenario, we may hypothesize that the lower MPOD responses in some participants in the present study may be related to the decrease of oxidative stress and endoplasmic reticulum signaling pathways, involving polymorphisms that require extensive research, as reported by our group regarding the effects of single nucleotide polymorphisms in vitamin $\mathrm{E}$ on serum biomarkers for ocular diseases [41].

Nevertheless, our findings highlight the relevance of LT availability and the adjunctive role of DHA when assessing the relevance of nutraceuticals in macular health and disease. Supporting this, previous reports emphasize the potential role of $\omega-3$ LC-PUFAs in late AMD prevention [42, 43]. Recently, Arunkumar et al.[33] confirmed the results from the AREDS2 supplement formulation 
[25] that oral formulations containing LT and ZX readily impact MPOD levels, lowering the rate of progression to advanced AMD and visual loss in the affected patients [44]. Furthermore, the Visucam ${ }^{\circledR}$ retinograph offered a suitable and relatively easy non-mydriatic tool for ocular fundus image and MPOD quantification for clinical studies.

Among the study limitations, the first is the relatively small sample size. Large cohorts have to be analyzed with a longer duration to better elucidate the role of LT and DHA in the healthy and pathologic macula. Second, our participants were selected with the intention of being well nourished, but tobacco habits were not taken into consideration. These 2 facts may partially interfere with the full generalizability of the results, especially when trying to extrapolate these data to AMD. In summary, taking altogether, our data strongly demonstrate that supplementation with daily oral doses of LT $(6 \mathrm{mg} /$ day) and DHA (700 mg/day) for 3 months is effective in increasing circulating LT levels as well as MPOD in healthy eyes.

\section{Acknowledgements}

The authors want to thank Carl Zeiss Meditec Iberia (Madrid, Spain) for lending the Visucam ${ }^{\circledR}$ retinograph during the research study course and to Brudylab (Barcelona, Spain) for kindly donating the 2 formulations utilized in the present study.

\section{Statement of Ethics}

The present study was conducted ethically in accordance with the World Medical Association Declaration of Helsinki. All subjects gave their written informed consent. The institute's Ethic Committee on human research approved the study protocol.

\section{Conflict of Interest Statement}

The authors report no conflicts of interest in this work.

\section{Funding Sources}

This work was partially funded by RETICs (OFTARED) from the Institute of Health Carlos III (Spanish Government) and by a grant from Brudylab ${ }^{\circledR}$ (Barcelona, Spain).

\section{Author Contributions}

Vicente Zanon-Moreno participated in work design and acquisition, analysis, and interpretation of data and also participated in the final approval of the version to be published. Joan C. DomingoPedrol participated in the acquisition, analysis, and interpretation of data for the work. Silvia M. Sanz-Gonzalez participated in the acquisition and interpretation of data for the work and also participated in the critical review of the draft version. Jorge RagaCervera participated in the acquisition of clinical data. Juan J. Salazar-Corral participated in the work design, in the critical review of the draft, and in the final approval of the version to be published. Maria D. Pinazo-Duran participated in the work design, acquisition of clinical data, in the critical review of the draft, as well as in the final approval of the version to be published.

\section{References}

1 McCusker MM, Durrani K, Payette MJ, Suchecki J. An eye on nutrition: the role of vitamins, essential fatty acids, and antioxidants in age-related macular degeneration, dry eye syndrome, and cataract. Clin Dermatol. 2016; 34(2):276-85.

2 Raman R, Vaghefi E, Braakhuis AJ. Food components and ocular pathophysiology: a critical appraisal of the role of oxidative mechanisms. Asia Pac J Clin Nutr. 2017; 26(4):572-85.

3 Walter RM, Uriu-Hare JY, Olin KL, Oster $\mathrm{MH}$, Anawalt BD, Critchfield JW, et al. Copper, zinc, manganese, and magnesium status and complications of diabetes mellitus. Diabetes Care. 1991 Nov;14(11):1050-6.

4 Vinetskaia MI, Iomdina EN. [Study of lacrimal fluid trace elements in several eye diseases]. Vestn Oftalmol. 1994 Oct-Dec;110(4): 24-6.
5 Gilbert R, Peto T, Lengyel I, Emri E. Zinc nutrition and inflammation in the aging retina. Mol Nutr Food Res. 2019 Aug;63(15): e1801049.

6 Johnson EJ. The role of carotenoids in human health. Nutr Clin Care. 2002;5(2):56-65.

7 White WS, Zhou Y, Crane A, Dixon P, Quadt F, Flendrig LM. Modeling the dose effects of soybean oil in salad dressing on carotenoid and fat-soluble vitamin bioavailability in salad vegetables. Am J Clin Nutr. 2017;106(4): 1041-51.

8 Johnson EJ, Neuringer M, Russell RM, Schalch W, Snodderly DM. Nutritional manipulation of primate retinas, III: effects of lutein or zeaxanthin supplementation on adipose tissue and retina of xanthophyll-free monkeys. Invest Ophthalmol Vis Sci. 2005; 46(2):692-702.
9 Bone RA, Landrum JT, Friedes LM, Gomez CM, Kilburn MD, Menendez E, et al. Distribution of lutein and zeaxanthin stereoisomers in the human retina. Exp Eye Res. 1997;64(2): 211-8.

10 Arteni AA, Fradot M, Galzerano D, MendesPinto MM, Sahel JA, Picaud S, et al. Structure and conformation of the carotenoids in human retinal macular pigment. PLoS One. 2015;10(8): $\mathrm{e} 0135779$.

11 Bartlett H, Howells O, Eperjesi F. The role of macular pigment assessment in clinical practice: a review. Clin Exp Optom. 2010;93(5): 300-8.

12 Ma L, Liu R, Du JH, Liu T, Wu SS, Liu XH Lutein, zeaxanthin and meso-zeaxanthin supplementation associated with macular pigment optical density. Nutrients. 2016;8(7): 426.
Optimized Nutraceutical Formulation with DHA on Macular Levels of Lutein
Ophthalmic Res 2021;64:1068-1076 DOI: $10.1159 / 000509439$ 
13 Gong Y, Fu Z, Liegl R, Chen J, Hellström A, Smith LE. $\omega-3$ and $\omega-6$ long-chain PUFAs and their enzymatic metabolites in neovascular eye diseases. Am J Clin Nutr. 2017;106(1):1626.

14 Assmann KE, Adjibade M, Hercberg S, Galan $\mathrm{P}$, Kesse-Guyot E. Unsaturated fatty acid intakes during midlife are positively associated with later cognitive function in older adults with modulating effects of antioxidant supplementation. J Nutr. 2018;148(12):1938-45.

15 Kouchaki E, Afarini M, Abolhassani J, Mirhosseini N, Bahmani F, Masoud SA, et al. High-dose omega-3 fatty acid plus vitamin D3 supplementation affects clinical symptoms and metabolic status of patients with multiple sclerosis: a randomized controlled clinical trial. J Nutr. 2018;148(8):1380-6.

16 Harris WS. The omega- 3 index as a risk factor for coronary heart disease. Am J Clin Nutr. 2008;87(6): 1997s-2002s.

17 Molfino A, Amabile MI, Mazzucco S, Biolo G, Farcomeni A, Ramaccini C, et al. Effect of oral docosahexaenoic acid (DHA) supplementation on DHA levels and omega-3 index in red blood cell membranes of breast cancer patients. Front Physiol. 2017;8:549.

18 Merle BM, Benlian P, Puche N, Bassols A, Delcourt C, Souied EH. Circulating omega-3 Fatty acids and neovascular age-related macular degeneration. Invest Ophthalmol Vis Sci. 2014;55(3):2010-9.

19 Johnson EJ, Chung HY, Caldarella SM, Snodderly DM. The influence of supplemental lutein and docosahexaenoic acid on serum, lipoproteins, and macular pigmentation. Am J Clin Nutr. 2008;87(5):1521-9.

20 Gasso F, Bogdanov P, Domingo JC. Docosahexaenoic acid improves endogen antioxidant defense in ARPE-19 cells. Invest Ophthalmol Vis Sci. 2008;49(13):5932.

21 J.C. D, Inventor. Use of DHA for treating a pathology associated with cellular oxidative damage. 2014.

22 Lafuente M, Ortín L, Argente M, Guindo JL, López-Bernal MD, López-Román FJ, et al. Three-year outcomes in a randomized singleblind controlled trial of intravitreal ranibizumab and oral supplementation with docosahexaenoic acid and antioxidants for diabetic macular edema. Retina. 2019;39(6): 1083-90.

23 Azar G, Quaranta-El Maftouhi M, Masella JJ, Mauget-Faysse M. Macular pigment density variation after supplementation of lutein and zeaxanthin using the Visucam ${ }^{\star} 200$ pigment module: impact of age-related macular degeneration and lens status. J Fr Ophtalmol. 2017;40(4):303-13.
24 Korobelnik JF, Rougier MB, Delyfer MN, Bron A, Merle BMJ, Savel H, et al. Effect of dietary supplementation with lutein, zeaxanthin, and omega- 3 on macular pigment: a randomized clinical trial. JAMA Ophthalmol. 2017;135(11):1259-66.

25 Colman-Martinez M, Martinez-Huelamo M, Miralles E, Estruch R, Lamuela-Raventos RM. A new method to simultaneously quantify the antioxidants: carotenes, xanthophylls, and vitamin A in human plasma. Oxid Med Cell Longev. 2015;2015:9268531.

26 Lepage G, Roy CC. Direct transesterification of all classes of lipids in a one-step reaction. J Lipid Res. 1986;27(1):114-20.

27 Garcia-Layana A, Recalde S, Alaman AS, Robredo PF. Effects of lutein and docosahexaenoic acid supplementation on macular pigment optical density in a randomized controlled trial. Nutrients. 2013;5(2):543-51.

28 Age-Related Eye Disease Study 2 Research Group. Lutein + zeaxanthin and omega- 3 fatty acids for age-related macular degeneration: the Age-Related Eye Disease Study 2 (AREDS2) randomized clinical trial. JAMA. 2013;309(19):2005-15.

29 Kaya S, Weigert G, Pemp B, Sacu S, Werkmeister RM, Dragostinoff N, et al. Comparison of macular pigment in patients with agerelated macular degeneration and healthy control subjects: a study using spectral fundus reflectance. Acta Ophthalmol. 2012;90(5): e399-403.

30 Mares JA, LaRowe TL, Snodderly DM, Moeller SM, Gruber MJ, Klein ML, et al. Predictors of optical density of lutein and zeaxanthin in retinas of older women in the Carotenoids in Age-Related Eye Disease Study, an ancillary study of the Women's Health Initiative. Am J Clin Nutr. 2006;84(5):1107-22.

31 Richer S, Devenport J, Lang JC. LAST II: differential temporal responses of macular pigment optical density in patients with atrophic age-related macular degeneration to dietary supplementation with xanthophylls. Optometry. 2007;78(5):213-9.

32 Olmedilla-Alonso B, Estévez-Santiago R, Silván J-M, Sánchez-Prieto $M$, de Pascual-Teresa S. Effect of long-term xanthophyll and anthocyanin supplementation on lutein and zeaxanthin serum concentrations and macular pigment optical density in postmenopausal women. Nutrients. 2018;10(8):959.

33 Arunkumar R, Calvo CM, Conrady CD, Bernstein PS. What do we know about the macular pigment in AMD: the past, the present, and the future. Eye. 2018;32(5):992-1004.
34 Connolly EE, Beatty S, Loughman J, Howard AN, Louw MS, Nolan JM. Supplementation with all three macular carotenoids: response, stability, and safety. Invest Ophthalmol Vis Sci. 2011;52(12):9207-17.

35 Kirby ML, Beatty S, Loane E, Akkali MC, Connolly EE, Stack J, et al. A central dip in the macular pigment spatial profile is associated with age and smoking. Invest Ophthalmol Vis Sci. 2010;51(12):6722-8.

36 Nolan JM, Akkali MC, Loughman J, Howard AN, Beatty S. Macular carotenoid supplementation in subjects with atypical spatial profiles of macular pigment. Exp Eye Res. 2012;101:9-15.

37 Obana A, Tanito M, Gohto Y, Okazaki S, Gellermann W, Bernstein PS. Changes in macular pigment optical density and serum lutein concentration in Japanese subjects taking two different lutein supplements. PLoS One. 2015; 10(10):e0139257.

38 Borel P. Genetic variations involved in interindividual variability in carotenoid status. Mol Nutr Food Res. 2012;56(2):228-40.

39 Feigl B, Morris CP, Voisey J, Kwan A, Zele AJ. The relationship between $\mathrm{BCMO} 1$ gene variants and macular pigment optical density in persons with and without age-related macular degeneration. PLoS One. 2014;9(2):e89069.

40 Meyers KJ, Johnson EJ, Bernstein PS, Iyengar SK, Engelman CD, Karki CK, et al. Genetic determinants of macular pigments in women of the Carotenoids in Age-Related Eye Disease Study. Invest Ophthalmol Vis Sci. 2013; 54(3):2333-45

41 Zanon-Moreno V, Asensio-Marquez EM, Ciancotti-Oliver L, Garcia-Medina JJ, Sanz P, Ortega-Azorin C, et al. Effects of polymorphisms in vitamin E-, vitamin C-, and glutathione peroxidase-related genes on serum biomarkers and associations with glaucoma. Mol Vis. 2013;19:231-42.

42 Delyfer MN, Buaud B, Korobelnik JF, Rougier MB, Schalch W, Etheve S, et al. Association of macular pigment density with plasma omega-3 fatty acids: the PIMAVOSA study. Invest Ophthalmol Vis Sci. 2012 Mar 9;53(3):120410.

43 Merle BM, Delyfer MN, Korobelnik JF, Rougier MB, Malet F, Féart C, et al. High concentrations of plasma $\mathrm{n} 3$ fatty acids are associated with decreased risk for late age-related macular degeneration. J Nutr. 2013;143(4):505-11.

44 Buscemi S, Corleo D, Di Pace F, Petroni M, Satriano A, Marchesini G. The effect of lutein on eye and extra-eye health. Nutrients. 2018; 10(9):1321. 